\title{
Problèmes hydrauliques liés au refroidissement des centrales nucléaires fonctionnant en circuit ouvert
}

\author{
Hydraulic problems comnected \\ with muclear power plant once-through cooling systems
}

\author{
Y. Coeffé et B. Manoha \\ Laboratoire national d'hydraulique \\ Electricité de France
}

\section{Introduction}

Les lois de la thermodynamique exigent la présence d'une "source froide" dans le cycle de fonctionnement d'une centrale nucléaire. Pour $1000 \mathrm{MW}$ d'électricité produite, $2000 \mathrm{MW}$ doivent ainsi être évacués pour refroidir le condenseur.

La solution la plus simple consiste à prélever un débit d'eau (prise) en mer ou en rivière et à le restituer avec un certain échauffement (rejet); c'est la solution du refroidissement en circuit ouvert.

Cette solution impose d'assurer la continuité d'un débit de refroidissement qui est très important, malgré diverses agressions du milieu (action de la houle et des courants, problèmes d'ensablement, d'envasement, de colmatage par des organismes marins, etc...) en limitant le plus possible l'impact sur le milieu (échauffement de l'eau, perturbation de l'équilibre sédimentologique du rivage, destruction des organismes marins, etc...), et le recyclage entre la prise et le rejet.

L'importance des débits mis en jeu conduit alors à tenir compte, au tout premier plan pour le choix d'un site en circuit ouvert, de la capacité du milieu à disperser les calories rejetées et de la possibilité de réaliser des ouvrages de prise et de rejet d'un coût acceptable et perturbant au minimum le milieu naturel.

Le but de cet article est de présenter les principaux problèmes hydrauliques liés au refroidissement d'une centrale nucléaire en circuit ouvert, que ce soit en site fluvial ou maritime, et de montrer les différents outils que l'on peut utiliser pour les aborder.

\section{Données du problème et critères à satisfaire}

Dans le cas de la réfrigération en circuit ouvert, les centrales nucléaires de forte puissance rejettent dans le milieu liquide de grosses quantités de chaleur: à titre d'exemple, pour une centrale de la filière eau légère produisant $5 \mathrm{GW}$ électriques, $10 \mathrm{GW}$ seront rejetés dans de l'eau réchauffée de 12 à $15^{\circ} \mathrm{C}$, et un débit de l'ordre de $200 \mathrm{~m}^{3} / \mathrm{s}$ est alors nécessaire (notons pour fixer les idées que ce débit correspond sensiblement au débit moyen de la Seine). La recherche de niveaux d'échauffement dans le milieu naturel aussi bas que possible requiert donc des débits de dilution des eaux de refroidissement très importants et oriente la prospection des sites vers le bord de mer, les estuaires et les grands fleuves capables d'assurer toute l'année un débit de refroidissement en circuit ouvert suffisant.

Le problème posé est alors celui du devenir de ces rejets thermiques, c'est-à-dire de la détermination de l'emprise de la "tache thermique" correspondant à la zone où la température de l'eau va être modifiée par les rejets d'eau chaude de la centrale. La connaissance de la tache thermique permet ensuite d'étudier les conséquences de ces rejets qui sont doubles. Tout d'abord il faut remarquer que du fait même de l'échauffement du milieu, l'eau captée à la prise pourra être à une température supérieure à la température "naturelle". On a donc une dégradation de la source froide et une baisse de rendement de la centrale; c'est ce qu'on appelle la "recirculation". Il y a ensuite les conséquences sur l'écologie du milieu.

Les problèmes hydrauliques liés à l'implantation des ouvrages de prise et de rejet, outre ceux liès à la tache thermique et à la recirculation, concernent d'autre part :

- l'impact des ouvrages sur les courants,

- les risques de gêne pour la navigation,

- l'impact sédimentologique éventuel et les modifications entraînées sur l'évolution des fonds, des rives ou du littoral

- la protection et le dimensionnement des ouvrages face à la houle, aux courants, aux effets météo, etc..

- le devenir des effluents radioactifs rejetés 
Les principaux critères à satisfaire, compte tenu des divers impératifs techniques et économiques, sont donc essentiellement :

- d'assurer en permanence un débit de refroidissement suffisant (problème de sûreté-circuit SEC)

- d'éviter une recirculation à la prise trop importante (problème d'efficacité et de rendement)

- de minimiser l'impact thermique (problème d'environnement)

La législation française, si elle fixe des normes de concentrations précises à ne pas dépasser pour les effluents radioactifs (arrêté du 10 août 1976 en particulier), impose simplement du point de vue impact thermique pour les sites en rivière une température maximale de $30^{\circ} \mathrm{C}$, les sites maritimes n'étant pas réellement soumis quant à eux à des normes précises; en fait chaque site est considéré comme un cas particulier pour lequel sont fixées des normes spécifiques.

\section{Choix des emplacements de prise et de rejet}

\subsection{Les phénomènes physiques}

\subsubsection{Le milieu naturel:}

Avant d'examiner les modifications du milieu dues à l'implantation des ouvrages de prise et de rejet, il convient d'examiner l'état "naturel" de celui-ci et de bien connaitre en particulier les paramètres qui vont influer sur la dilution des calories, c'est-à-dire essentiellement les vents, les courants, les débits fluviaux pour un site en rivière, la température naturelle du milieu.

Cette température varie à la fois dans l'espace et dans le temps. En ce qui concerne le milieu marin en particulier, on constate une assez grande régularité des températures en moyenne annuelle sur de très longues périodes, mais par contre il existe des variations des moyennes mensuelles d'une année à l'autre au gré des aléas météorologiques. Ceci est encore plus vrai à l'échelle plus fine des semaines ou des jours. Au voisinage des côtes cette variation peut être encore rendue plus complexe (en particulier par d'éventuels rejets côtiers naturels ou non).

A ces hétérogénéités horizontale et temporelle de température, il faut ajouter, en général, une hétérogénéité verticale. Celle-ci vient du fait que les variations de température du milieu marin sont dues aux échanges de chaleur avec l'atmosphère par rayonnement, convection et évaporation, et que ceux-ci modifient d'abord la

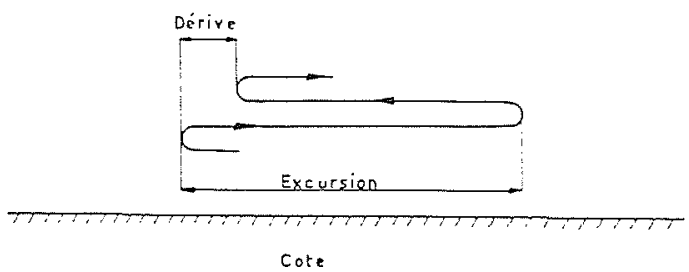

Figure 1 - Schématisation du phénomène "Upwelling".

couche superficielle de la mer. C'est ensuite la diffusion verticale, due en particulier à la turbulence, qui assure l'évolution de la température des eaux les plus profondes. Ces phénomènes peuvent entraîner, dans les mers où les courants sont peu importants et donc de diffusion verticale faible, une véritable stratification en deux couches: une couche de surface, plus chaude donc plus légère, et une couche de fond plus froide de quelques degrés (fig. 1). Ce fait est en particulier observé dans la mer Méditerranée pendant tout l'été, la séparation entre les deux couches (la thermocline) évoluant par des profondeurs de 30 à $50 \mathrm{~m}$.

Les mouvements des masses d'eau résultent de mécanismes fort complexes. En se limitant à la zone littorale qui nous intéresse ici, nous pouvons considérer que les causes essentielles des courants sont la marée et le vent. Les courants de marée, essentiellement périodiques, ont une origine astronomique et sont liés aux variations du niveau de la mer dues à la marée. Ils se caractérisent par une bonne uniformité verticale.

Au voisinage des côtes, ces courants sont grossièrement parallèles à celles-ci, et les masses d'eau sont entraînées, au cours d'une marée, d'un côté puis de l'autre sur plusieurs kilomètres (de l'ordre d'une quinzaine par exemple sur les côtes françaises le long de la Manche): c'est "l'excursion" (voir fig. 2). Mais au bout d'un cycle de marée, les masses d'eau n'ont pas exactement repris leur position initiale : la distance apparente parcourue est désignée sous le nom de "dérive". Ainsi un point du littoral ne verra pas toujours passer les mêmes eaux puisque la dérive les entraînera progressivement hors du champ d'observation, et plus la dérive sera grande, plus vite les eaux seront renouvelées devant le site considéré.

Dans les mers à marée très faible, le vent prend beaucoup d'importance dans la création des courants. Ceux-ci ont alors une répartition verticale non uniforme due à l'action conjuguée de la contrainte qui s'exerce en surface et de la force de Coriolis. Ces courants, d'intensité assez modeste (de quelques $\mathrm{cm} / \mathrm{s}$ à quelques dizaines de $\mathrm{cm} / \mathrm{s}$ ) ont une variation aléatoire dans le temps. On

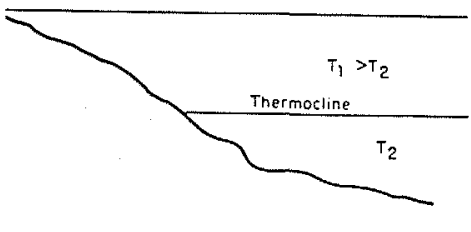

Avant le soup de vent

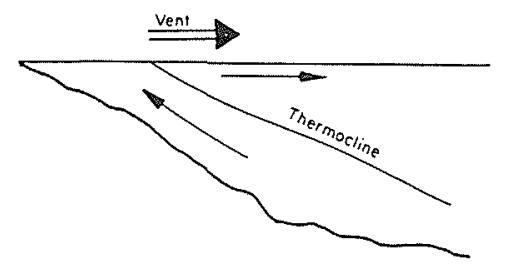

Pendont le coup de vent

Figurc 2 - Schématisation des phénomènes "Excursion" et "Dérive". 
peut, comme pour les courants de marée, mais par intégration sur une certaine période de temps, définir un courant de dérive. Dans ces mers à marée faible, apparaît souvent en été le phénomène, déjà mentionné plus haut, de thermocline. On peut alors observer, lors de forts coups de vent, un basculement de celle-ci et l'apparition à la côte des eaux profondes, plus froides (fig. 1). Ces remontées ou "upwelling" expliquent certaines variations brutales de température qu'on peut observer.

En rivière les phénomènes saisonniers sont fondamentaux et conditionnent les régimes de débit (crues, étiages) et de température naturelle.

\subsubsection{Les mécanismes de dilution}

\section{A) Rejet en mer}

a. La dilution dans le champ proche des ouvrages de rejet:

La formation de la tache thermique en mer fait intervenir non seulement plusieurs phénomènes différents mais aussi des couplages entre ceux-ci. Examinons tout d'abord ces phénomènes pris isolément.

En partant de l'ouvrage de rejet, nous observons en premier lieu une dilution de ce jet d'eau échauffée par l'eau du milieu ambiant, elle-même mise en mouvement par les courants marins; c'est ce que nous appelons le champ proche. Cette zone a une emprise relativement faible (de l'ordre de quelques $\mathrm{km}^{2}$ ) mais voit la température chuter très rapidement de la température de l'eau rejetée à une température élevée de quelques degrés tout au plus au-dessus de la température naturelle du milieu.

La dilution dans cette zone est due à l'entrâtnement dans le jet du fluide ambiant plus froid. La dynamique d'un jet est un problème bien connu en mécanique des fluides, au moins dans le cas du jet homogène dans un milieu au repos. On distingue classiquement une zone dite potentielle, où la vitesse et la concentration restent constantes sur l'axe du jet, puis une zone d'écoulement établi dans laquelle vitesse et concentration décroissent. Les profils transversaux de ces grandeurs ont des allures gaussiennes (cf. fig. 3).

Dans le problème qui nous intéresse ici, celui des rejets d'eau chaude sur le littoral, la physique est plus complexe puisqu'interviennent aussi les forces de gravité (l'eau rejetée est plus légère) et l'existence d'un courant dans le milieu récepteur, mais les caractéristiques générales données plus haut sur le comportement d'un

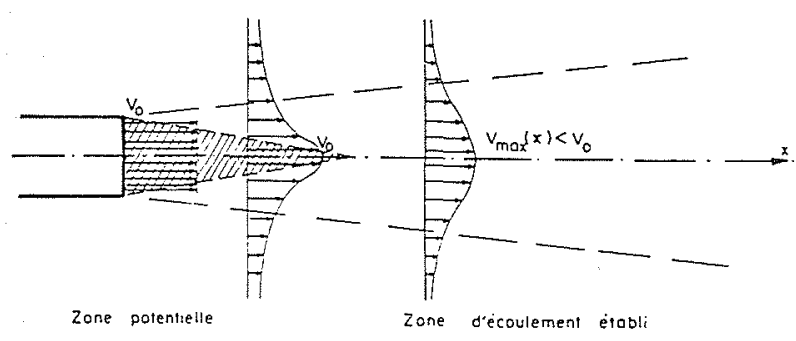

Figure 3. - Profils transversaux d'un jet de fluide dans un milieu ambiant plus froid. jet restent valables. On observe simplement que le jet d'eau chaude va avoir tendance à venir "flotter" en surface et par suite à s'élargir dans le plan horizontal. L'expérience montre en outre que la présence d'un courant traversier accélère de façon très importante la dilution dans le champ proche.

La dilution dans ce champ proche dépend, c'est évident, beaucoup des ouvrages de rejet.

Disons simplement ici qu'une dilution très rapide sera obtenue en fractionnant le débit de rejet (diffuseur multipores) et en restituant celui-ci dans le milieu marin à des vitesses élevées (plusieurs $\mathrm{m} / \mathrm{s}$ ). Un rejet en canal par contre donnera une dilution plus lente.

Si la trajectoire du jet passe au voisinage de l'ouvrage de prise, l'eau aspirée par celui-ci va être déjà échauffée : on parle alors de recirculation directe. Les conséquences de celle-ci sont néfastes, aussi bien pour le rendement de la centrale que pour l'écologie ; pour celle-ci notamment l'échauffement à la sortie du condenseur, par rapport au milieu naturel, qui sera la somme de la valeur de cette recirculation (qui peut atteindre plusieurs ${ }^{\circ} \mathrm{C}$ ) et de l'échauffement au condenseur, peut conduire à des températures létales pour certaines espèces. On doit donc s'attacher à bien disposer l'ouvrage de prise pour rendre minimale cette recirculation.

\section{b. Le champ lointain:}

L'effet de jet va en diminuant lorsqu'on s'éloigne de l'ouvrage de rejet et les masses d'eau échauffées sont alors prises en charge par les courants marins. Ceux-ci vont transporter et disperser ces calories qui passeront ensuite dans l'atmosphère. Ce dernier point mérite d'être bien mis en valeur et retenu: c'est en définitive dans l'atmosphère que passeront les calories rejetées à la mer qui ne feront que transiter dans celle-ci.

Dans cette zone, qu'on appelle le champ lointain, interviennent donc trois phénomènes: la convection, la diffusion-dispersion et les échanges mer-atmosphère.

Le phénomène de transport-convection n'appelle guère de commentaire car il est suffisamment intuitif. Rappelons cependant les notions d'excursion et de dérive (fig. 2) définies plus haut et qui interviennent ici. Le phènomène de diffusion est déjà beaucoup plus complexe et fait intervenir en particulier ce qu'on appelle la turbulence des écoulements et qui est la cause d'échanges beaucoup plus importants dans une masse d'eau en mouvement que dans une masse d'eau au repos, et où n'interviendrait que la diffusion moléculaire. La diffusion moléculaire et la diffusion turbulente sont créées par des mouvements désordonnés, au niveau de la molécule dans le premier cas et au niveau de la particule d'eau (qui contient un grand nombre de molécules) dans le second cas.

Le phénomène de diffusion turbulente pose encore bien des problèmes aux chercheurs mais l'ingénieur a assez d'informations à son sujet pour en tenir compte, d'une façon approchée certes mais suffisante, dans les problèmes d'évaluation de tache thermique.

Les échanges atmosphériques constituent le puits des calories. La puissance $P(T)$ échangée par unité de surface à travers un plan d'eau à la température $T$, résulte des échanges par convection, rayonnement et évaporation. On appelle température d'équilibre $T_{e q}$, celle 
pour laquelle cette puissance est nulle. En linéarisant autour de cette température, on obtient une expression simple de la puissance échangée, en introduisant un coefficient $A$ qu'on appelle coefficient d'échanges atmosphériques:

$$
P(T)=A\left(T-T_{e q}\right)
$$

La valeur de ce coefficient permet en particulier d'évaluer l'échelle des temps caractéristiques des échanges atmosphériques dans la formation de la tache thermique. On trouve un temps de l'ordre d'une à deux semaines.

Les courants marins vont donc transporter et disperser ces calories mais, en raison de leur direction variable dans le temps, vont créer, au niveau même des ouvrages de prise et de rejet, un échauffement par rapport à la température naturelle: c'est ce que nous appellerons le champ de température de base.

Ceci entraîne deux conséquences. L'une pour l'ouvrage de prise: cet échauffement résiduel crée un phénomène de recirculation qu'on appelle dans ce cas différée pour la distinguer de la recirculation directe. La deuxième conséquence concerne l'ouvrage de rejet: l'eau rejetée va se diluer dans un milieu déjà échauffé.

En résumé, on peut donc dire que tout se passe comme si on diluait une eau échauffée du $\Delta T$ condenseur augmenté de la recirculation (différée et éventuellement directe) dans l'eau des courants marins échauffée à la température de base. Un schéma de la formation de la tache thermique est donné sur la figure 4.

\section{B) Rejet en rivière}

Le rejet dans la rivière de l'eau échauffée engendre deux phénomènes distincts: un mélange en aval et, éventuellement, un coin de densité vers l'amont.

a) Champ thermique aval, le mélange

Deux zones sont encore à distinguer:

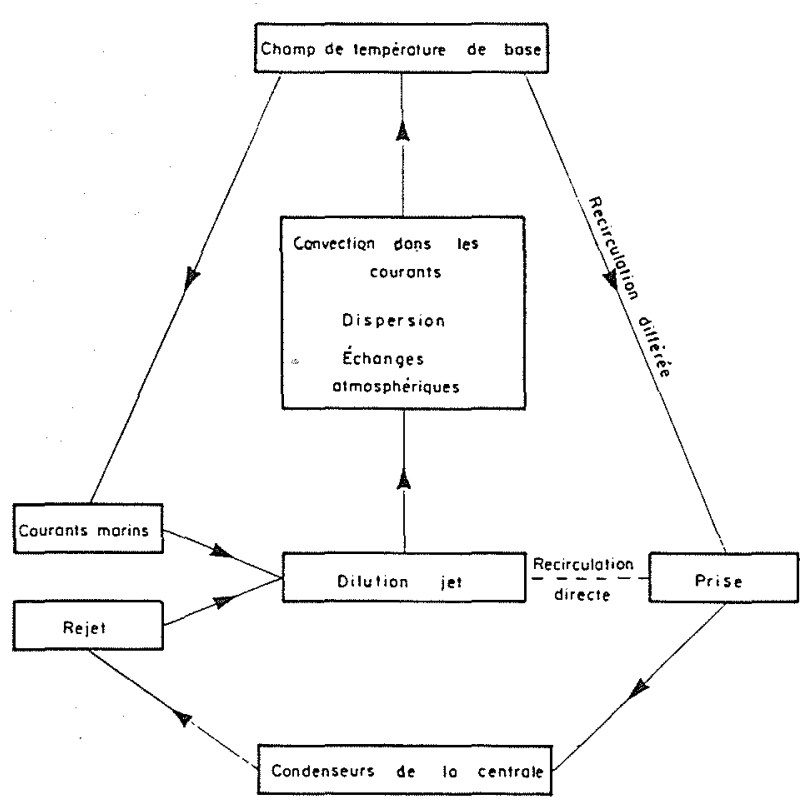

Figure 4. - Schéma de formation de la tache thermique.
- au voisinage immédiat du rejet, la zone dite du champ proche où les conditions de mélange dépendent directement des caractéristiques du cours d'eau et des ouvrages de rejet: débits des eaux chaudes et froides, leurs vitesses et températures respectives ;

- plus en aval, la zone du champ lointain où l'évolution des températures dépend principalement des phénomènes de diffusion, de convection et d'échanges atmosphériques.

\section{b) Champ thermique amont, la recirculation}

Quand le débit du cours d'eau est nettement supérieur au débit dérivé, la totalité des eaux échauffées est évacuée vers l'aval. Par contre, en période d'étiage, l'eau chaude peut former un coin de densité qui remonte dans le cours d'eau.

La longueur du coin chaud dépend des conditions de rejet, du rapport des débits et des vitesses, de l'élévation de température au rejet ainsi que de la valeur du coefficient de frottement au fond.

Lorsque la longueur du coin est supérieure à la distance prise-rejet, une fraction de l'eau échauffée pénètre dans la prise, entrainant une recirculation des eaux de refroidissement.

\subsection{Choix des emplacements de prise et de rejet}

\subsubsection{La prise}

Par définition l'ouvrage de prise doit se situer dans une zone où l'alimentation en eau reste assurée quelles que soient les conditions naturelles, en particulier les décotes dues aux hautes pressions atmosphériques ou aux vents de terre pour un site marin, et les débits d'étiage pour les sites en rivière ; il convient d'autre part d'éviter les zones d'ensablement ou cette alimentation pourrait être compromise à la longue ou en cas d'apport brutal par une tempête ou une crue soudaine.

\subsubsection{Le rejet}

Le choix de la position du rejet répond avant tout au souci d'assurer la meilleure dispersion des calories récupérées dans les condenseurs : afin d'optimiser la dilution initiale il importe que ce rejet se situe dans un espace le moins confiné possible (rejet au large par grands fonds); la dispersion dans l'écoulement ambiant sera ensuite fonction de l'intensité des courants, de la dérive et de la turbulence ainsi que du degré d'uniformité de cet écoulement.

\subsubsection{Dispositif relatif prise-rejet}

La disposition relative de la prise et du rejet doit être déterminée de façon à éviter autant que possible le retour des eaux chaudes à la prise, phénomène que l'on appelle recirculation ou recyclage (fig. 5) et qui est particulièrement nuisible puisqu'il réduit le rendement de la centrale et qu'il augmente l'échauffement au rejet et donc l'impact sur l'environnement écologique.

La disposition optimale dépend en fait essentiellement des conditions de débits fluviaux pour un site en rivière et de la courantologie sur le site, et éventuellement de la topographie des fonds et de la géométrie de la côte pour les sites en bord de mer. 




$0)$ Directe.

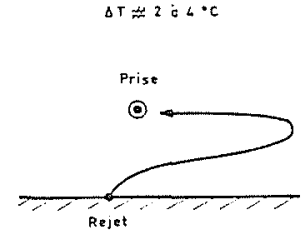

b) Indirecte

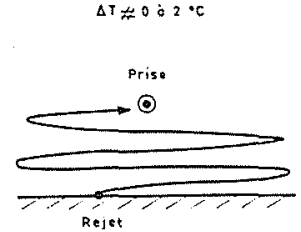

c) Differé

Figure 5. - Schématisation du phénomène de "Recirculation".

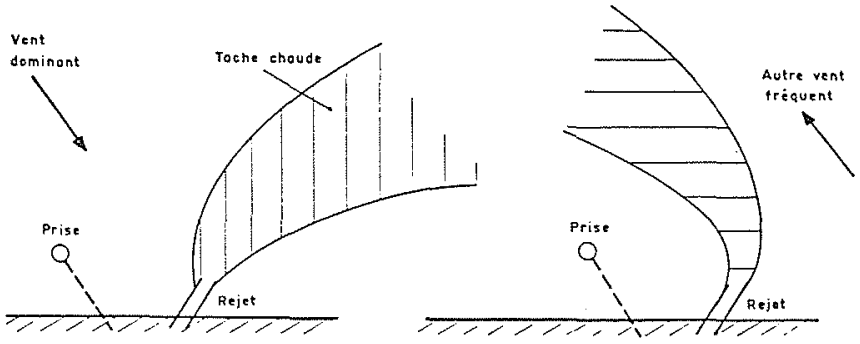

Figure 6. - Dispositition "Prise-Rejet" en mer sans marée.

Dans le cas où le sens de l'écoulement est invariable, ou lorsqu'il existe un courant dominant, il y a tout intérêt à situer la prise à l'amont du rejet par rapport à cet écoulement (rivières non soumises à la marée); de même dans les mers sans marée où le cheminement des eaux rejetées est essentiellement fonction des caractéristiques du vent, il est souhaitable de placer la prise à l'amont du rejet par rapport aux vents dominants (fig. 6). Enfin dans le cas des mers à marée les courants alternatifs véhiculent les eaux chaudes de part et d'autre $\mathrm{du}$ rejet sur toute la longueur de l'excursion (distance parcourue par une particule d'eau pendant le flot ou le jusant): cependant ces courants gardent généralement une direction constante, sensiblement parallèle à la côte, si bien que l'éloignement de la prise par rapport au rejet dans le sens perpendiculaire à la côte (fig. 7) permet d'éviter la recirculation directe (transport direct de l'eau rejetée vers la prise).

L'utilisation de la topographie des fonds ou de l'existence d'obstacles (naturels ou artificiels) à l'écoulement sera illustrée plus loin par l'exemple des centrales du Blayais et de Gravelines.

\subsection{Choix du type d'ouvrages}

Il convient de distinguer les circuits de prise et de rejet et leurs ouvrages d'extrémité.

Les circuits de prise et de rejet peuvent fonctionner soit en charge, soit à surface libre. Le circuit en charge
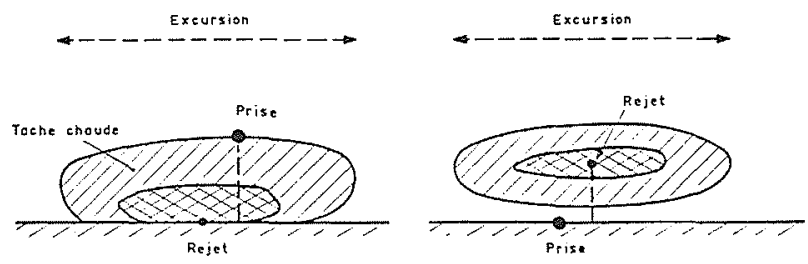

Figure 7. - Disposition "Prise-Rejet" en mer à marée. (galerie souterraine ou conduite ensouillée avec fortes vitesses de rejet) est de réalisation et d'entretien délicats mais son impact thermique sur le milieu est très limité; par contre, il peut poser des problèmes de stabilité du lit ou de navigation, en particulier en rivière.

Le circuit à surface libre est beaucoup plus facile de réalisation et de surveillance mais en bord de mer il est plus vulnérable et son impact sur l'environnement n'est plus négligeable ; c'est pourquoi il ne sera généralement retenu que pour les ouvrages pour lesquels cet impact reste limité.

Les ouvrages d'extrêmité sont essentiellement caracrérisés par deux paramètres :

- profondeur d'aspiration et de rejet

- courants (vitesse et cap) qu'ils induisent à proximité immédiate.

Pour la prise le choix de la profondeur d'aspiration, compte tenu de l'impératif majeur qui est d'assurer le débit de refroidissement quelles que soient les circonstances, est fonction en mer de la houle, du niveau minimal du plan d'eau, des risques d'aspiration des sédiments du fond et de la couche d'eau que l'on souhaite aspirer (sélection en température ou teneur en organismes vivants); en rivière, il est parfois nécessaire de construire un seuil pour assurer une hauteur d'eau suffisante même dans des conditions d'étiage (Saint Laurent-des-Eaux, Dampierre). La vitesse d'aspiration doit être déterminée en fonction des risques d'aspiration des organismes vivants; le courant d'aspiration doit être également aussi horizontal que possible, les poissons résistant très mal aux vitesses verticales. La prise d'eau s'effectue donc soit par l'intermédiaire d'un avant-port ou d'un canal qui devra, en particulier en mer, être protégé contre la houle avec des vitesses d'aspiration faibles mais avec des risques d'absorption des pollutions de surface et des sédiments du fond, soit grâce à des ouvrages en forme de tulipe évasée (pour obtenir des vitesses horizontales) et des canalisations en charge permettant d'aller chercher l'eau au large. 
Quant à l'ouvrage de rejet, il doit assurer une dilution aussi efficace que possible des eaux chaudes dans le milieu ambiant, en particulier dans la zone proche où l'échauffement est le plus important: il est donc souhaitable d'effectuer ce rejet par forte vitesse dans un milieu non confiné, donc suffisamment loin de la surface et du fond. Les études menées sur les différents sites en bord de mer ont conduit à effectuer le rejet soit en canal près du rivage, ce qui impose des vitesses au rejet assez faibles (de l'ordre de $50 \mathrm{~cm} / \mathrm{s}$ ) et donc un mélange médiocre, soit par galerie immergée rejetant horizontalement au large avec des vitesses plus fortes dans une direction opposée à la prise. L'exemple de Paluel permet d'illustrer ces choix (voir plus loin). En rivière les rejets de surface en canal orienté vers l'aval sont les plus classiques, bien que moins favorables à une bonne dilution que les rejets de fond à grande vitesse moins couramment utilisés pour des raisons essentiellement de navigation.

\section{Moyens d'étude}

L'application des considérations théoriques précédemment exposées à un site particulier nécessite en premier lieu une bonne connaissance du milieu; il est donc indispensable d'entreprendre très tôt les mesures in situ concernant les débits fluviaux, la houle, les courants, la marée, le transport de sédiments, d'étudier les mouvements du rivage et de procéder à la reconnaissance des fonds (bathymétrie et nature du sol). Ces mesures sont utilisées à tous les stades de l'élaboration du projet; leur simple interprétation sur plans permet d'effectuer les études préliminaires; par la suite elles servent de référence d'étalonnage (marée, courants) ou de données de base (houle, bathymétrie) aux modèles mathématiques et physiques utilisés au stade de l'avant-projet et du projet définitif.

\subsection{Etudes préliminaires}

Les études préliminaires sur plans ou à l'aide de modèles numériques simples, concernent principalement l'impact thermique de la centrale; elles permettent également de définir une première disposition de la prise et du rejet à partir de l'analyse des données de courants et de dérives.

\subsection{Etudes d'avant-projet}

Les études d'avant-projet concernent les impacts courantologiques et thermiques de la centrale ainsi que la définition des ouvrages d'eau; elles sont effectuées à l'aide de différents outils :

- études sur plans quant à la détermination des structures d'ouvrages telles que les digues ou les protections

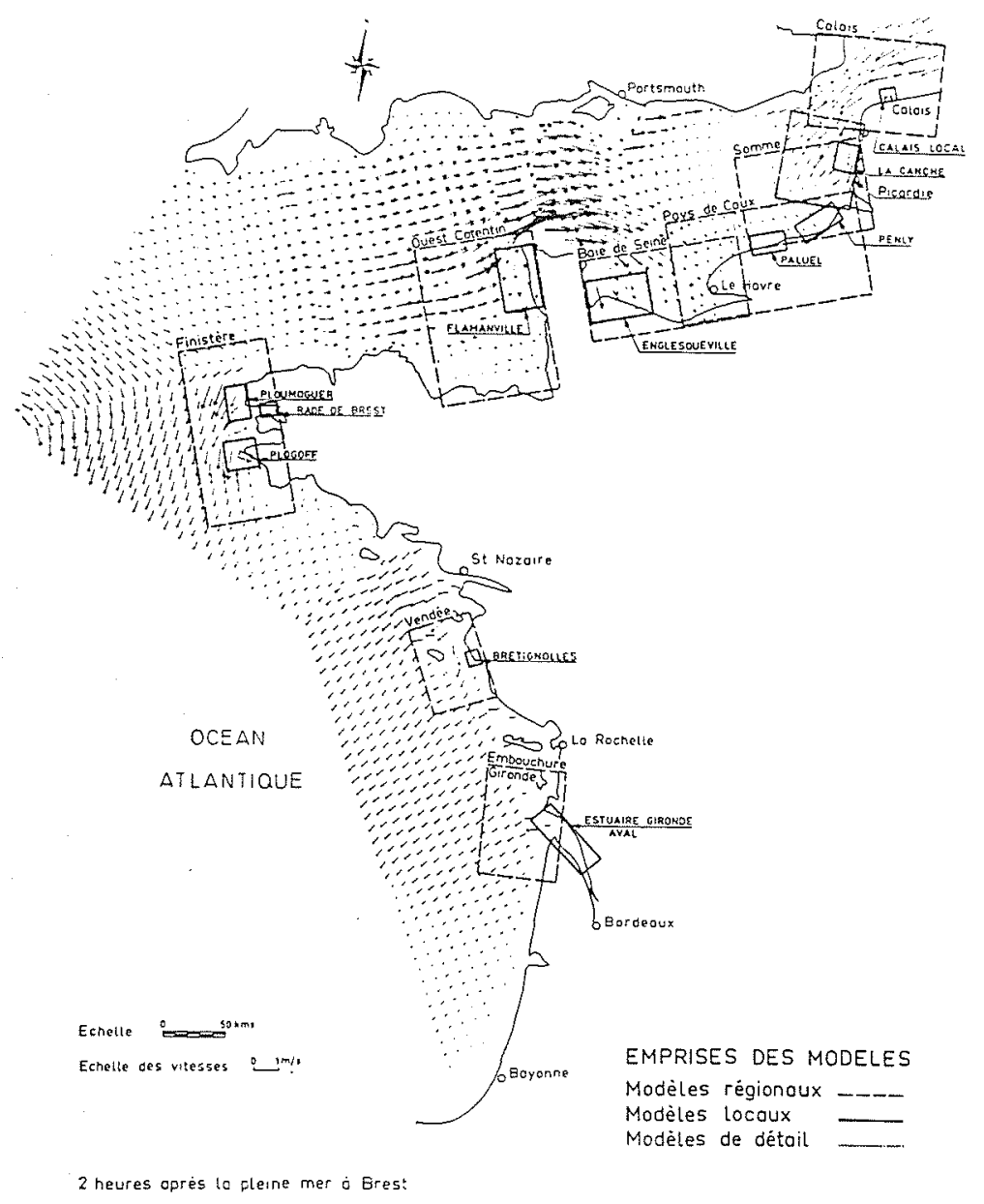

Figure 8. - Courants de marée sur les côtes de France. 


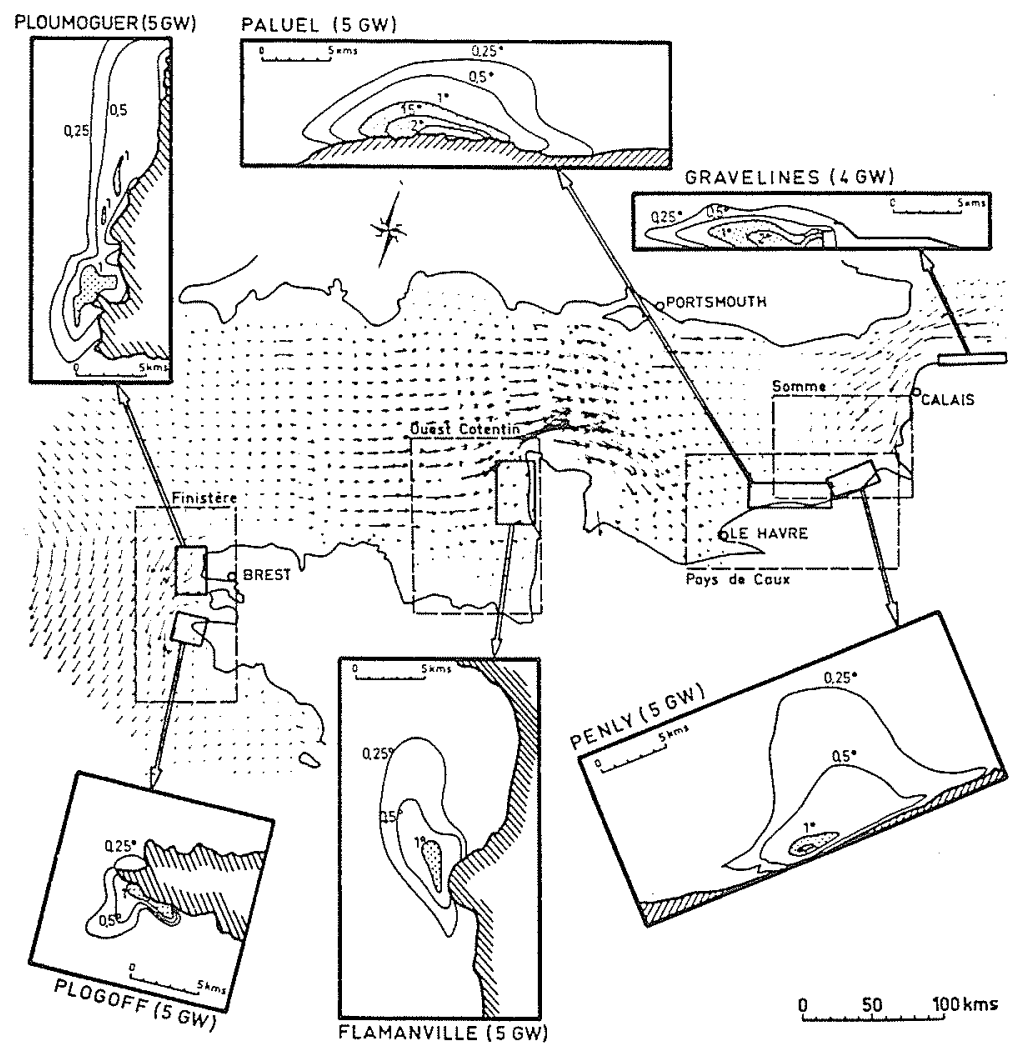

Figure 9. - Taches thermiques calculées pour les centrales électro-nucléaires en projet en France sur les côtes de la Manche.

des éventuels remblais gagnés sur la mer pour asseoir la centrale (Flamanville, Penly) :

- étude sur modèle mathématique de la courantologie au droit du site (fig. 8), des remontées du coin chaud en site en rivière (choix des distances minimales priserejet), de la propagation de la houle au large et à l'intérieur des ouvrages à surface libre (en vue de déterminer les caractéristiques de la houle au pied des ouvrages et les valeurs de l'agitation à l'intérieur), de la dilution au rejet et du champ thermique proche et lointain (fig. 9 et 10).

- étude sur modèle réduit à grande échelle des ouvrages immergés afin de déterminer le champ de vitesse induit au voisinage (prise ou rejet), l'éventuelle apparition de vortex, la répartition du débit entre les différents orifices (prise) ainsi que la dilution initiale des eaux chaudes (rejet).

\subsection{Etudes de projet}

Les études de projet sont par contre exclusivement effectuées sur modèles réduits; elles concernent:

- l'affinage du tracé des ouvrages à surface libre (minimisation de l'agitation et qualité de l'écoulement) et la mise au point de leur structure (essais de stabilité en canal puis en cuve à houle) ;

- la disposition relative de la prise et des différents rejets en vue de réduire au minimum les surfaces occupées par les forts isoéchauffements ainsi que la recirculation directe (modèle réduit à l'échelle du $1 / 100^{\mathrm{e}}$ au $1 / 250^{\mathrm{e}}$ );
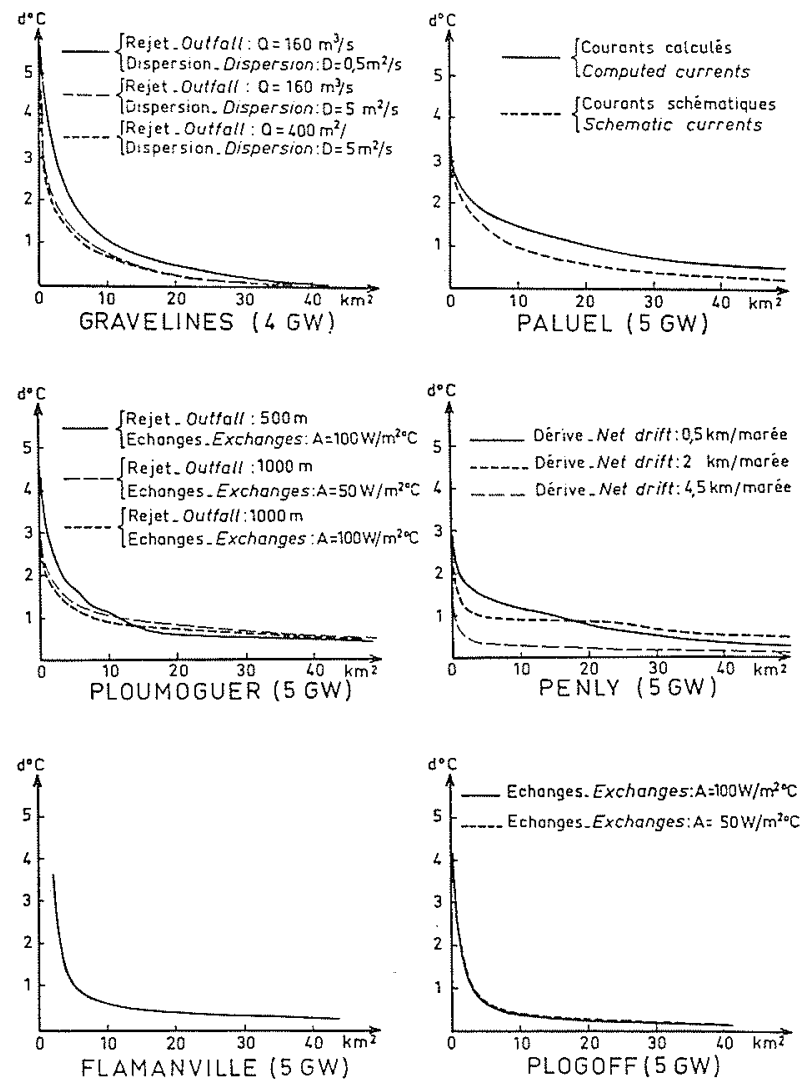

Figure 10. - Surfaces moyennes échauffées en fonction de l'augmentation de la température ambiante. 
- la stabilité des ouvrages immergés et les mesures d'efforts sur les structures en fonction des caractéristiques de la houle et des courants;

- l'impact sédimentologique lié à la présence des ouvrages et aux débits qui y transitent, surtout dans le cas de fonds sableux ou vaseux (le Blayais, Gravelines, sites en rivière) ou lorsque le littoral ou la rivière est bordé de sédiments soumis à un transit que risquent d'interrompre les ouvrages (galets de Paluel et de Penly).

Enfin, selon les sites examinés, des problèmes particuliers peuvent apparaître (colmatage des filtres par les algues ou par les détritus flottants, aspiration de bancs de poissons ou de nappes d'hydrocarbure, risques de destruction de la prise par des navires désemparés en mer, etc.).

Le tableau I indique, à titre d'exemple, les études effectuées pour les centrales de Paluel et de Flamanville.

Tableau I

\begin{tabular}{|l|l|}
\hline \multicolumn{1}{|c|}{ Type d'étude } & \multicolumn{1}{|c|}{ Objet } \\
\hline Mesures en nature & $\begin{array}{l}\text { Température - Bathymétrie - Marée - } \\
\text { Houle - Courants }\end{array}$ \\
\hline Etudes sur plan & $\begin{array}{l}\text { Impact thermique - Positionnement } \\
\text { initial de la prise et du rejet - Structure } \\
\text { des ouvrages. }\end{array}$ \\
\hline $\begin{array}{l}\text { Etudes sur modèle } \\
\text { mathématique }\end{array}$ & $\begin{array}{l}\text { Réfraction de la houle - Agitation - } \\
\text { Courantologie - Champ thermique } \\
\text { proche - Champ thermique lointain - } \\
\text { Recirculation - Détermination statis- } \\
\text { tique des houles et surcotes extrêmes - } \\
\text { Efforts dus à la houle sur les ouvrages } \\
\text { de rejet. }\end{array}$ \\
\hline $\begin{array}{l}\text { Etudes sur modèle } \\
\text { physique }\end{array}$ & $\begin{array}{l}\text { Dilution au rejet - Champ de courant } \\
\text { à la prise (tulipe) - Formation de } \\
\text { vortex - Agitation devant les stations } \\
\text { de pompage - Ecoulement dans le canal } \\
\text { de prise - Stabilité des digues là 2 et } \\
\text { 3 dimensions) - Stabilité de l'ouvrage } \\
\text { de rejet - Champ thermique proche - } \\
\text { Recirculation directe. }\end{array}$ \\
\hline
\end{tabular}

\subsection{Développement de techniques nouvelles}

L'affinage de certaines études délicates, telles que la détermination du champ thermique proche, a conduit à élaborer des modèles mathématiques adaptés à ces problèmes et à perfectionner la technique du modèle physique.

L'étude du positionnement des différents rejets de la centrale de Paluel a été menée simultanément sur modèle mathématique et sur modèle physique et est en cours actuellement pour Penly. Le but de cette étude est de déterminer la disposition mutuelle optimale des rejets au large permettant, tout en limitant la longueur des galeries de liaison, de réduire l'intéraction des panaches issus des différents rejets, particulièrement pendant les phases de courant établi pendant lesquelles le champ thermique proche varie peu. Les calculs ont été effectués sur un modèle tridimensionnel résolvant en régime permanent l'équation de Navier-Stokes couplée à l'équation de continuité et à l'équation de la chaleur ; ce modèle, valable uniquement pour le champ proche, s'applique lorsque les courants ambiants ne sont pas trop faibles (supérieurs à $1 / 5$ environ de la vitesse au rejet).

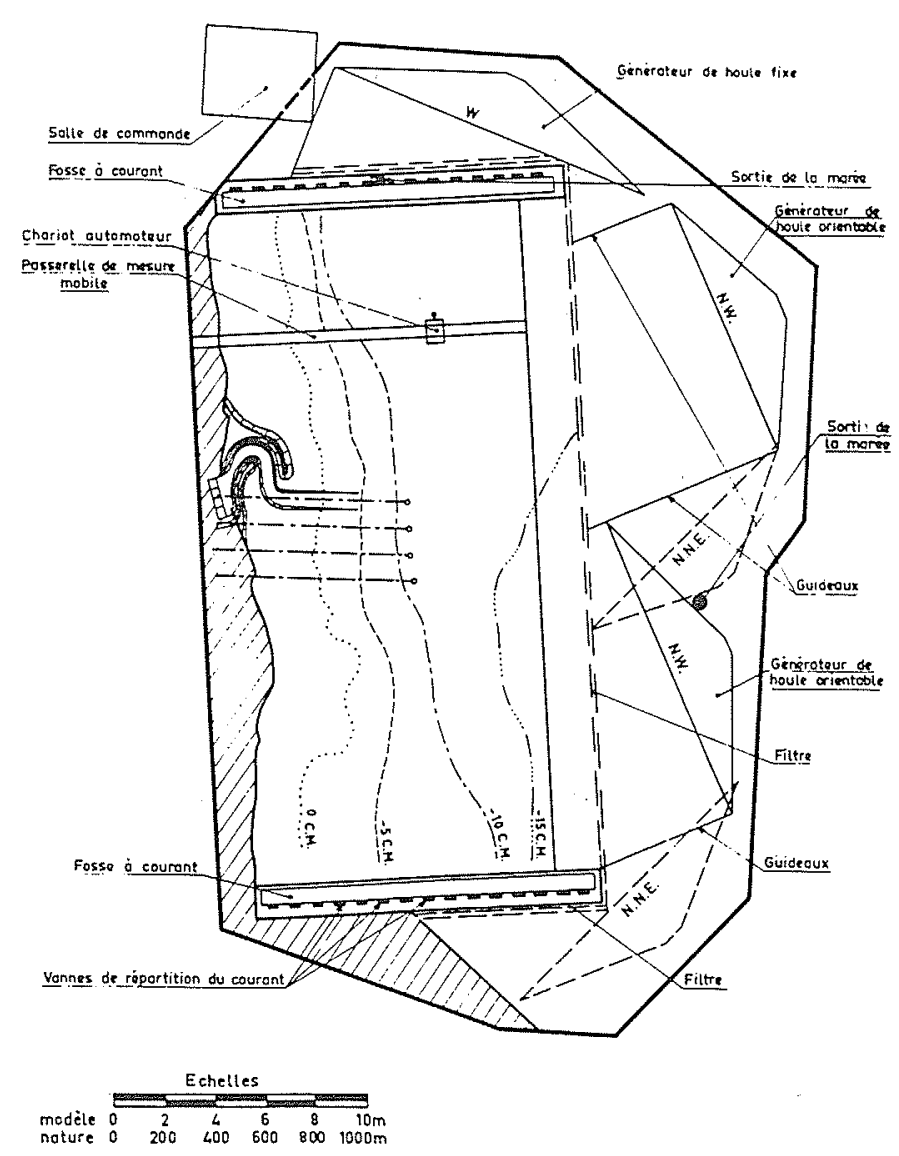

Figure 11. - Centrale du Paluel-Plan du modèle réduit

Le modèle physique, à l'échelle du $1 / 100^{\mathrm{e}}$ (fig. 11) représente environ $5 \mathrm{~km}^{2}$ de frange côtière; sur ce modèle sont reproduits la marée, les courants, la houle et le débit d'eau aspiré par la centrale et rejeté après échauffement de $15^{\circ} \mathrm{C}$; l'acquisition et le traitement automatiques des mesures de températures permettent l'établissement de cartes d'échauffement. Leur comparaison avec les résultats du calcul (fig. 12) permet le calage du modèle mathématique. Les calculs sont alors effectués pour diverses configurations des rejets (fig. 13), dont les plus favorables sont ensuite étudiées en détail sur le modèle physique. Le principal enseignement fourni par cette étude est qu'au niveau des forts échauffements l'intéraction de deux rejets ne peut être évitée si ceux-ci sont alignés dans la direction du courant, quelle que soit leur distance, cependant qu'un faible écartement dans la direction perpendiculaire à l'écoulement suffit à en dissocier les effets.

La centrale de Flamanville ( 4 tranches de $1300 \mathrm{MW}$ ) située dans le Cotentin prend l'eau nécessaire au refroidissement de ses circuits par l'intermédiaire d'un chenal à la côte protégé par des digues, et la rejette au large par des galeries forées. L'importance des ouvrages a conduit à utiliser des modèles mathématiques et physiques 

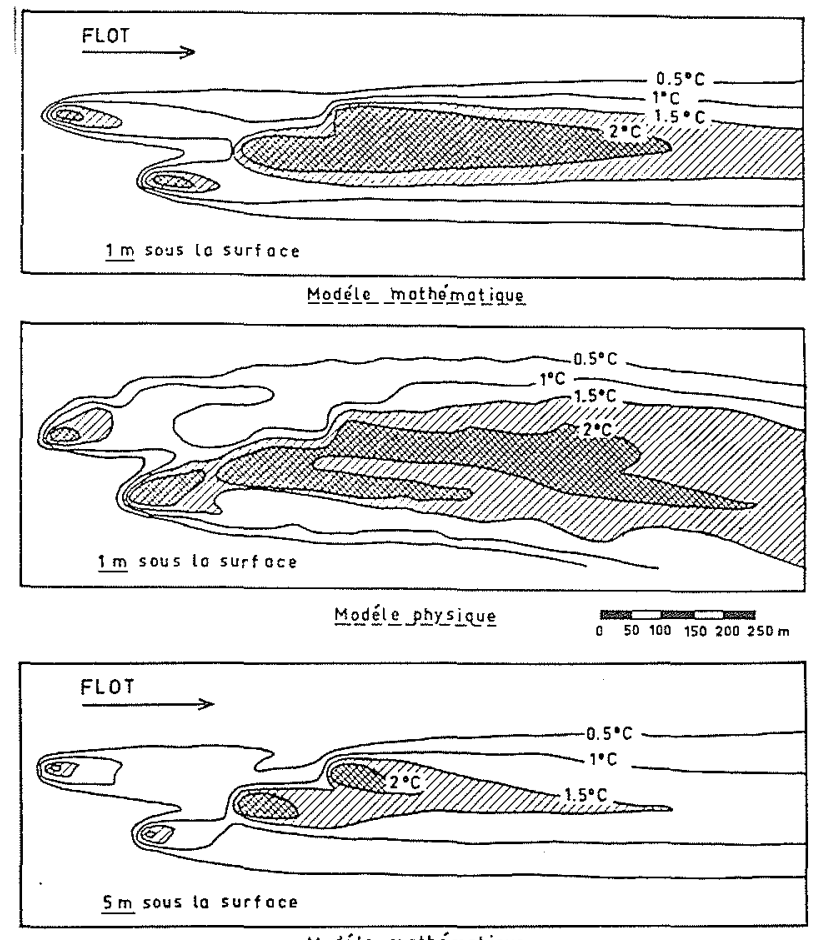

Modéle-mathématique

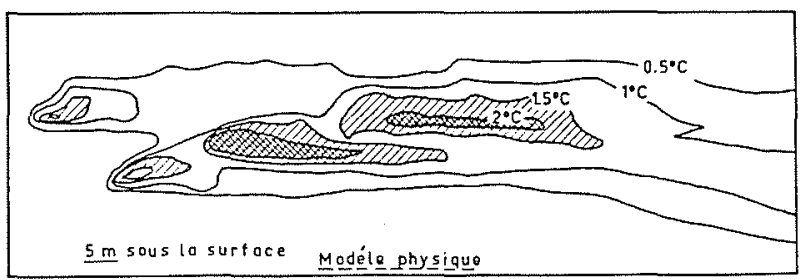

Figure 12. - Centrale de Paluel - Echauffements en plein flot à différentes profondeurs. Comparaison des modèles mathématique et physique.

permettant d'estimer les courants sur le site et l'impact de la centrale sur l'environnement. A cet effet, on utilise en particulier une cuve courantologique pilotée par calculateur (fig. 14) : ce modèle fonctionnant comme un banc d'essai, permet à partir de conditions limites issues d'un modèle mathématique de courants, et reproduites par un système de pompage et de réglage de niveau contrôlés par calculateur, d'estimer l'impact courantologique et thermique de la centrale dans le champ proche, et de minimiser la recirculation en optimisant la position des différents rejets. L'utilisation de ce modèle hybride, d'un type nouveau, combinant modèle physique et modèle mathématique, rassemble les avantages des deux techniques en éliminant les inconvénients majeurs que sont la schématisation du maillage mathématique et la durée très importante de l'étalonnage d'un modèle réduit classique. Sur le site de Flamanville la cuve courantologique a permis, en particulier, de reproduire fidèlement les courants de retour dans les anses au Nord et au Sud du cap où est située la centrale, courants qui sont d'une importance primordiale pour la recirculation.
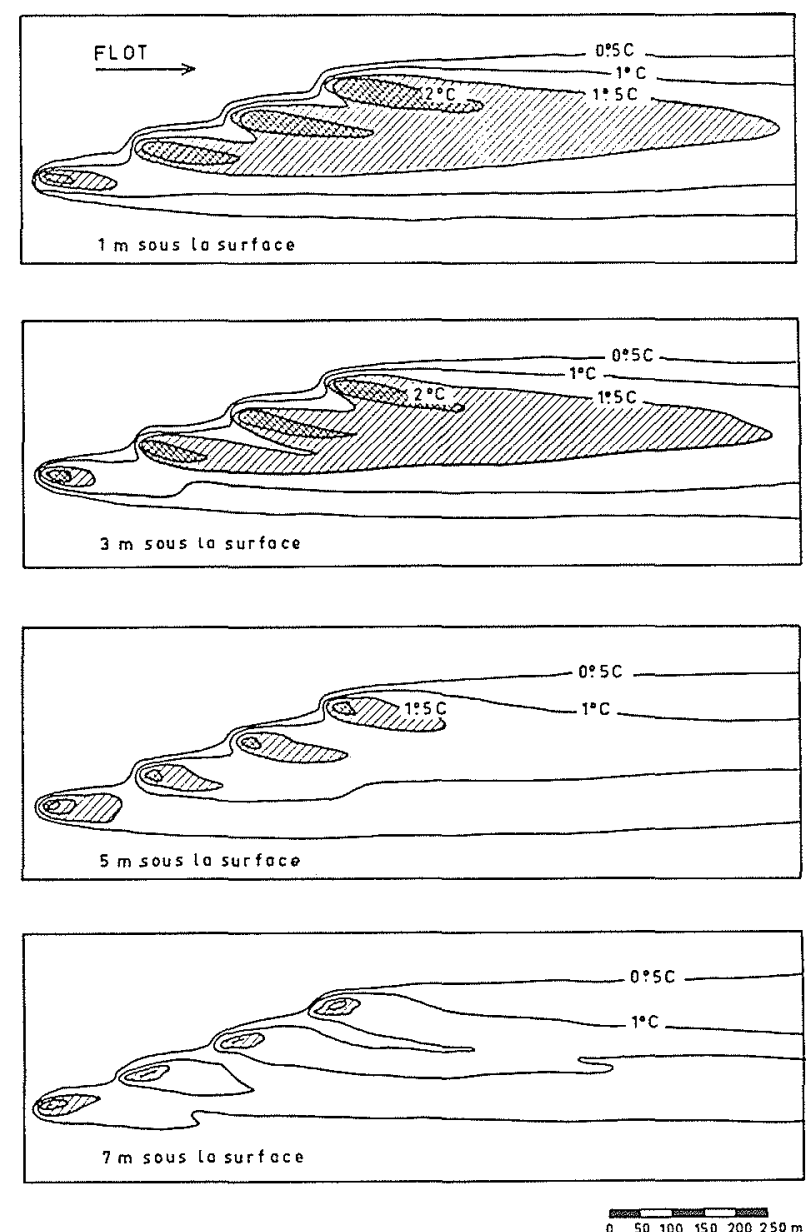

Figure 13. - Centrale de Paluel - Projet final - Echauffements calculés pour différentes profondeurs en flot

\section{Exemples}

- L'exemple de Gravelines (fig. 15) permet d'illustrer le cas de l'utilisation d'un port: la prise de la centrale est située au fond du nouvel avant-port de Dunkerque, dans une zone abritée de la houle et où les sédiments sont peu mobiles; le rejet des eaux chaudes s'effectue en haut de plage à l'Ouest du port; l'écran constitué par la digue Ouest du port permet alors d'éviter toute recirculation directe. En contrepartie cette digue crée une zone d'eaux mortes au niveau du rejet; la dilution y est alors assez faible et les eaux chaudes ont tendance à s'accumuler à l'abri de la digue où elles ont tendance à flotter en surface avant d'être prises par les courants du large où le mélange se fait plus efficacement (fig. 16).

- La centrale de Paluel est implantée en bord de Manche dans le Pays de Caux. Le schéma initial du circuit de refroidissement (fig. 17) prévoyait de prélever l'eau froide au large par fonds de $10 \mathrm{~m}$ sous les plus basses mers au moyen de "tulipes" permettant d'obtenir des vitesses d'aspiration faibles et horizontales; cette eau était ensuite, après échauffement dans les condenseurs, rejetée par un canal à surface libre de $50 \mathrm{~m}$ de large et $600 \mathrm{~m}$ de long. 


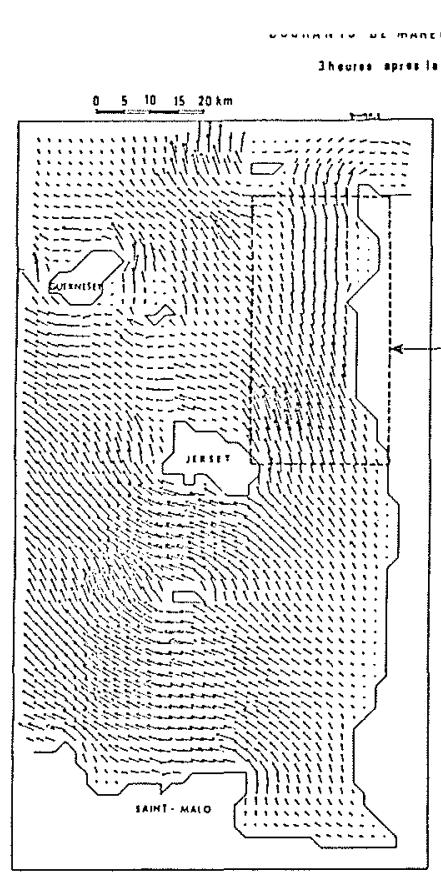

MODELE REGIONAL
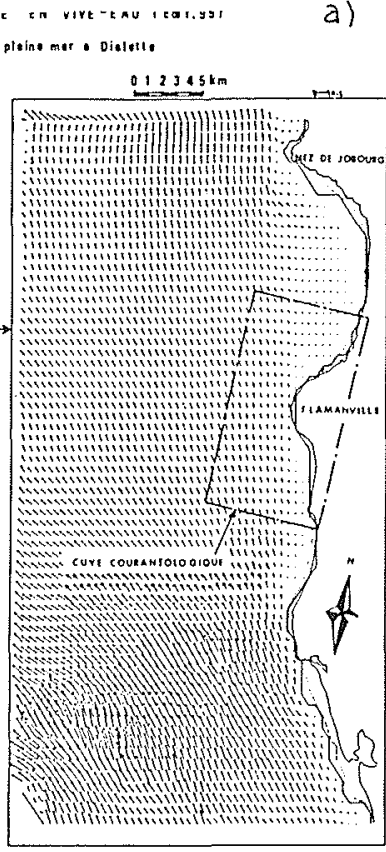

MODELE LOCAL

a)

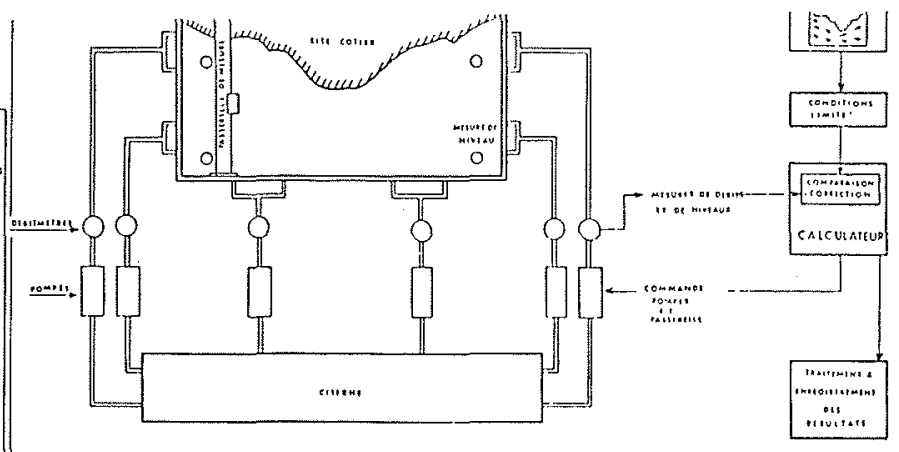

b)

Figure 14. - Centrale de Flamanville.

a) Emprise des modèles numériques de courants et de la cuve courantologique.

b) Cuve courantologique - Schéma de principe du système de pilotage

c) Cuve courantologique - Courants et échauffements obtenus in situ 4 heures avant la pleine mer.

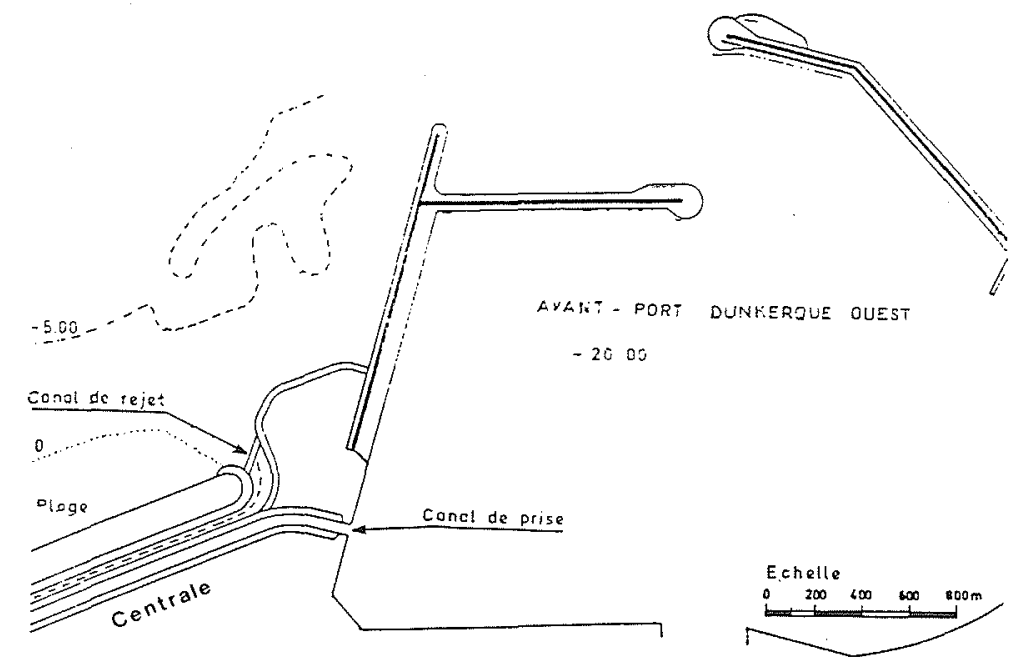

Figure 15. - Centrale de Gravelines - Ouvrages de prise et de rejet.

Ce dispositif a été abandonné au profit du suivant (fig. 18): l'eau froide est amenée aux stations de pompage par un canal à surface libre creusé dans l'estran et dont le tracé sinueux entre deux digues en enrochements est destiné à amortir la houle avant les stations de pompage; les eaux chaudes sont rejetées à $800 \mathrm{~m}$ de la falaise, au niveau des anciennes prises, à des vitesses de l'ordre de $3 \mathrm{~m} / \mathrm{s}$.

Cette seconde solution a pour principaux avantages une plus grande sûreté et un entretien plus facile des circuits de prise d'eau, une minimisation des pertes de charge à l'aspiration (donc un calage moins bas des stations de pompage), et une meilleure dilution initiale des eaux chaudes rejetées à grande vitesse réduisant du même coup la recirculation.

En contrepartie la seconde solution impose la construction d'ouvrages de protection des stations de pompage et peut éventuellement poser des problèmes de 
Figure 16. - Centrale de Gravelines - Panache de flot - Thermographie aérienne du 2 juin 1981 à 10 h 30 (pleine mer)
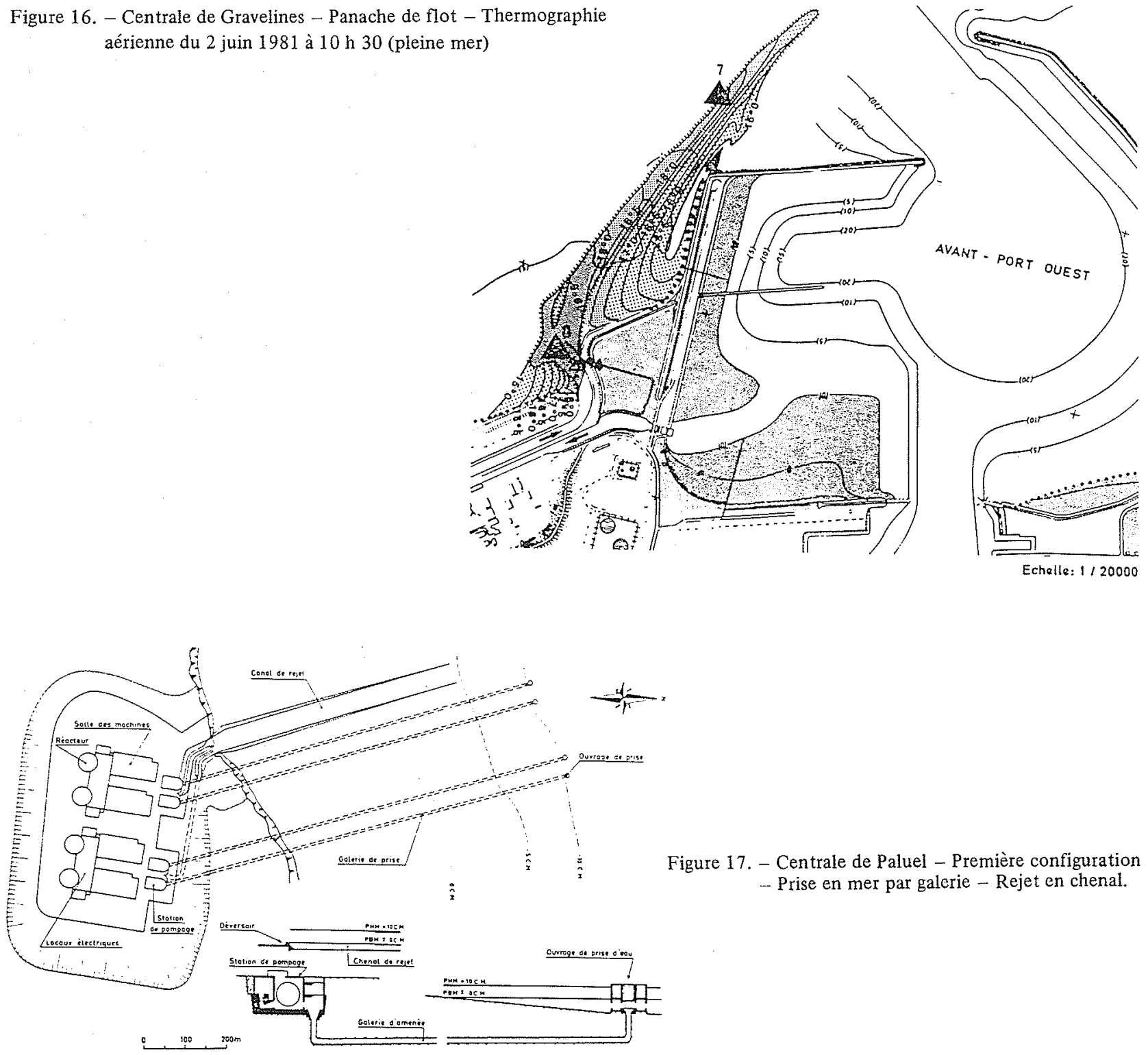

Figure 17. - Centrale de Paluel - Première configuration - Prise en mer par galerie - Rejet en chenal.

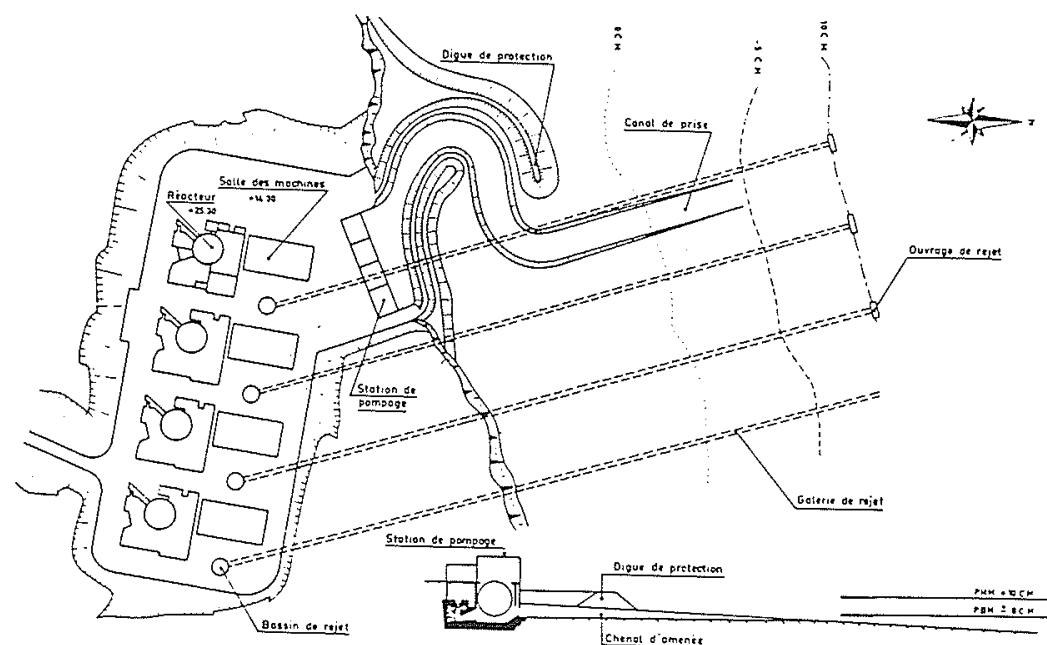

Figure 18. - Centrale de Paluel - Deuxième configuration - Prise en chenal - Rejet au large par galerie.

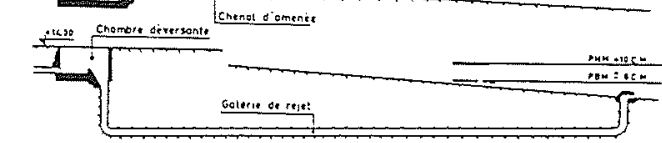


sédimentation dans les ouvrages de prise. Les calculs d'impact thermique dans le champ moyen et lointain sont présentés (fig. 19).

- La centrale du Blayais est située sur la rive droite de l'estuaire de la Gironde, à $50 \mathrm{~km}$ de l'embouchure. Les différents dispositifs de prise et de rejet en rive droite conduisaient à une recirculation importante due aux courants de marée alternatifs et au faible mélange transversal.

La géométrie particulière de l'estuaire a permis d'envisager une solution plus satisfaisante (fig. 20): au droit de la centrale, l'estuaire est divisé en trois chenaux séparés par deux bancs de sable parallèles à la rive; les chenaux canalisent l'écoulement et les bancs de sable réduisent le mélange transversal, si bien que les eaux chaudes issues du rejet restent en grande partie confinées dans le chenal où elles sont déversées. La prise en compte de ce phénomène a conduit à la solution suivante: la prise est située en rive droite à $300 \mathrm{~m}$ de la rive et le rejet dans le chenal central; les deux ouvrages sont reliés à la centrale par des conduites ensouillées afin de ne pas faire obstacle à la remontée des larves d'anguille et au mouvement des sédiments.

- Dans le cas de sites en rivière plusieurs systèmes peuvent être utilisés pour minimiser l'impact thermique et assurer la continuité du débit de refroidissement; citons en particulier l'emploi d'un seuil barrant la rivière pour avoir toujours une hauteur d'eau suffisante à la prise (Saint Laurent-des-Eaux, Dampierre), le rejet de fond implanté dans le seuil lui-même (Dampierre), l'emploi de bassins annexes pour minimiser les pro. blèmes de recirculation (Cattenon, Tricastin fig. 21).
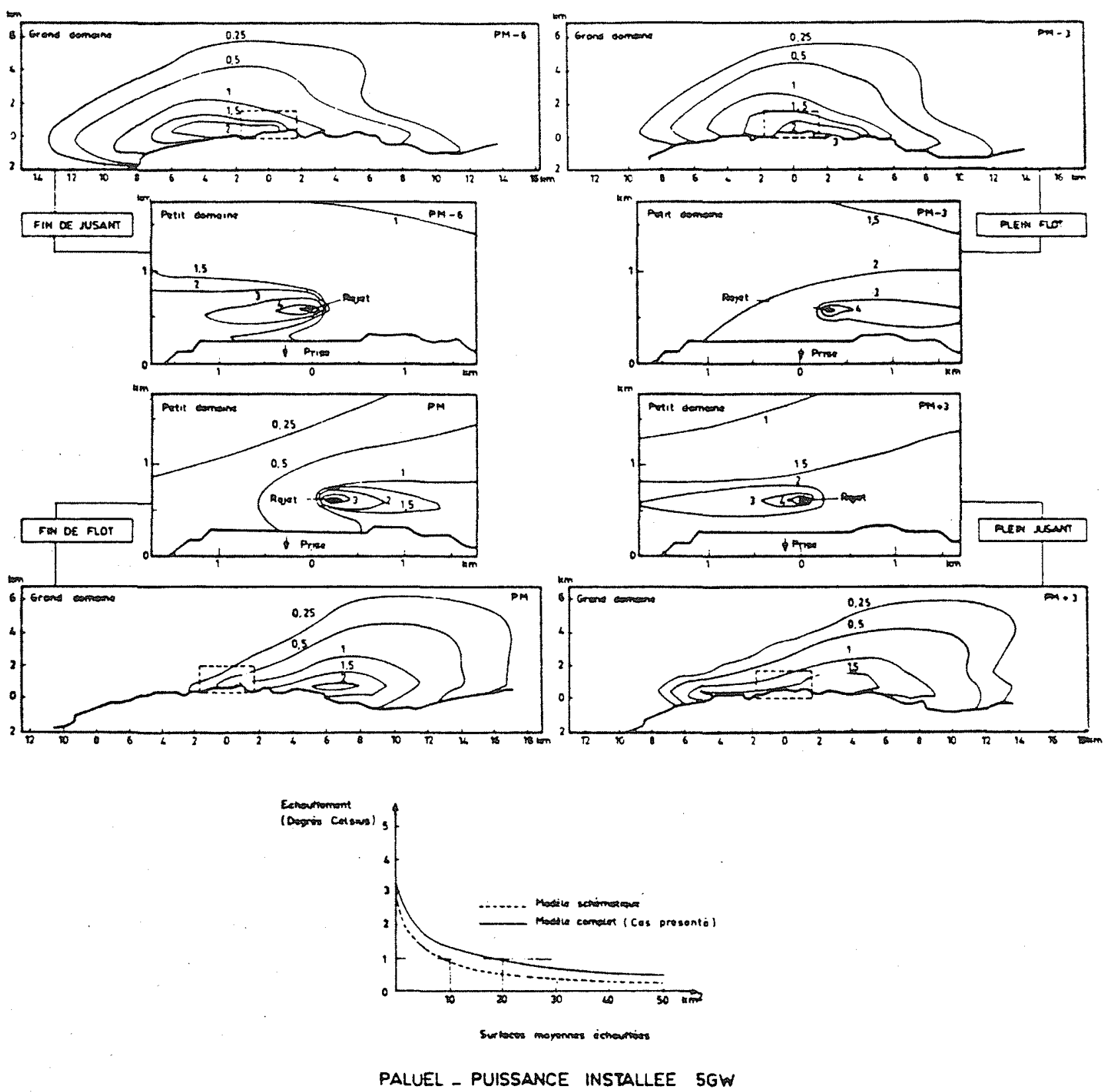

Figure 19. - Centrale de Paluel - Calculs d'impact thermique dans le champ moyen et le champ lointain 


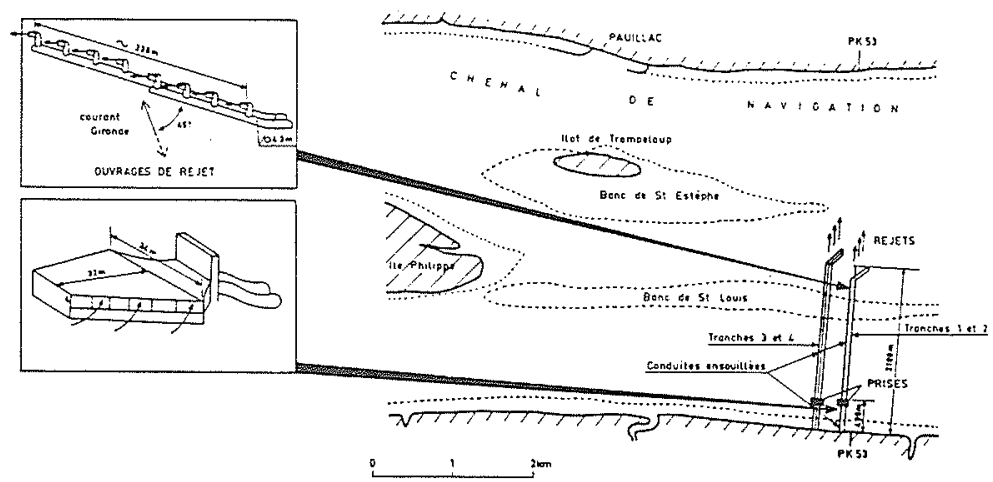

Figure 20. - Centrale duBlayais - Disposition retenue pour les prises et les rejets.

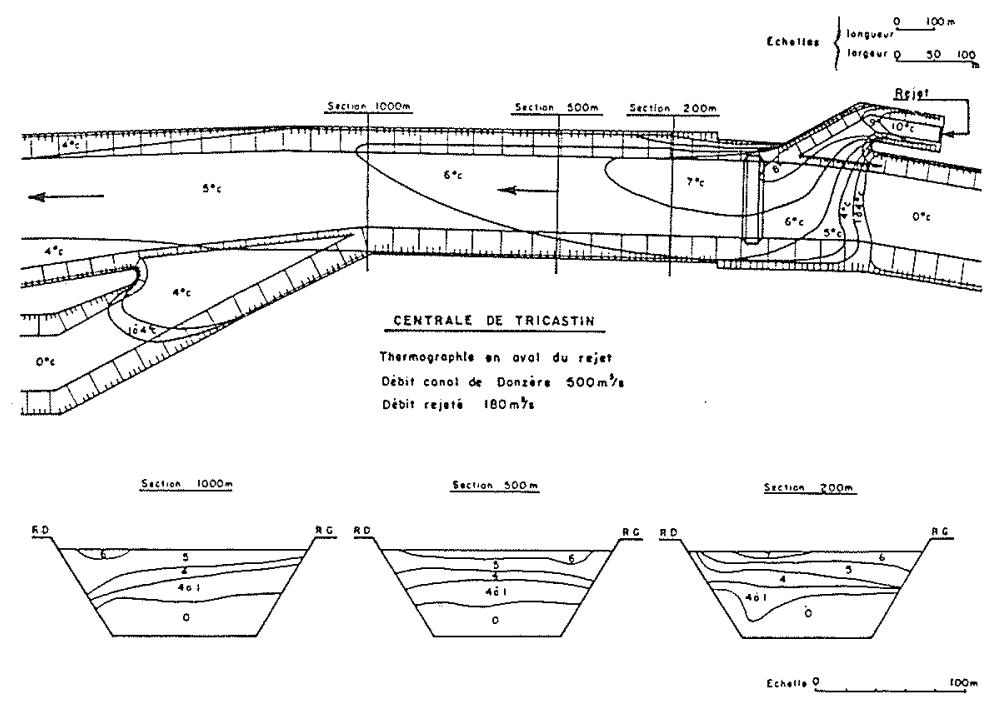

Figure 21. - Centrale du Tricastin - Thermographie en aval du rejet.

Débit du canal de Donzère : $500 \mathrm{~m}^{3} / \mathrm{s}$

Débit reje té

$: 180 \mathrm{~m}^{3} / \mathrm{s}$

\section{Conclusion}

La conception des ouvrages d'eau des centrales nucléaires a posé des problèmes nouveaux par l'importances des débits mis en jeu: conception d'ouvrages d'une ampleur inaccoutumée, dispersion de la chaleur et des effluents. De plus, la sûreté nucléaire oblige à garantir une alimentation en eau quelles que soient les circonstances, d'où la conception d'ouvrages particuliers.

La nouveauté et l'importance de ces problèmes ont amené l'élaboration de méthodologies nouvelles.

L'expérience acquise permet actuellement de traiter de nombreuses questions grâce aux progrès réalisés tant du point de vue modélisation physique que mathématique.

$\mathrm{Ce}$ n'est cependant que lorsque les premières centrales de cette nouvelle génération seront entrées en fonctionnement qu'il sera possible d'effectuer des mesures nature pour vérifier et affirner les modèles utilisés (cas de Gravelines en particulier où des études dites de "suivi" sont en cours actuellement). 